MATHEMATICS OF COMPUTATION

Volume 76, Number 259, July 2007, Pages 1639-1660

S 0025-5718(07)01961-8

Article electronically published on January 10, 2007

\title{
ON UNIVOQUE PISOT NUMBERS
}

\author{
JEAN-PAUL ALLOUCHE, CHRISTIANE FROUGNY, AND KEVIN G. HARE
}

\begin{abstract}
We study Pisot numbers $\beta \in(1,2)$ which are univoque, i.e., such that there exists only one representation of 1 as $1=\sum_{n>1} s_{n} \beta^{-n}$, with $s_{n} \in$ $\{0,1\}$. We prove in particular that there exists a smallest univoque Pisot number, which has degree 14. Furthermore we give the smallest limit point of the set of univoque Pisot numbers.
\end{abstract}

\section{INTRODUCTION}

Representations of real numbers in non-integer bases were introduced by Rényi 27. and first studied by Rényi and by Parry [26, 27]. Among the questions that were addressed is the uniqueness of representations. Given a sequence $\left(s_{n}\right)_{n \geq 1}$, Erdös, Joó and Komornik, 20], gave a purely combinatorial characterization for when there exists $\beta \in(1,2)$ such that $1=\sum_{n \geq 1} s_{n} \beta^{-n}$ is the unique representation of 1 . This set of binary sequences is essentially the same as a set studied by Cosnard and the first author [1, 2, 4] in the context of iterations of unimodal continuous maps of the unit interval.

Following [22, 23, a number $\beta>1$ is said to be univoque if there exists a unique sequence of integers $\left(s_{n}\right)_{n \geq 1}$, with $0 \leq s_{n}<\beta$, such that $1=\sum_{n \geq 1} s_{n} \beta^{-n}$. (Note that we consider only the representation of 1 . The uniqueness of the representation of real numbers in general was studied in particular in 21.) Using the characterization of 20, Komornik and Loreti constructed in 22] the smallest real number in $(1,2)$ for which 1 has a unique representation. Its representation happens to be the famous Thue-Morse sequence (see for example [5]).

Are there univoque Pisot numbers? It is worth noting that if the base $\beta$ is the "simplest" non-integer Pisot number, i.e., the golden ratio, then the number 1 has infinitely many representations. In this paper we study the univoque Pisot numbers belonging to $(1,2)$. We prove in particular (Theorem [5.4) that there exists a smallest univoque Pisot number, and we give explicitly the least three univoque Pisot numbers in $(1,2)$ : they are the roots in $(1,2)$ of the polynomials

$$
\begin{array}{ll}
x^{14}-2 x^{13}+x^{11}-x^{10}-x^{7}+x^{6}-x^{4}+x^{3}-x+1 & (\text { root } \approx 1.8800), \\
x^{12}-2 x^{11}+x^{10}-2 x^{9}+x^{8}-x^{3}+x^{2}-x+1 & (\text { root } \approx 1.8868), \\
x^{4}-x^{3}-2 x^{2}+1 & (\text { root } \approx 1.9052) .
\end{array}
$$

Received by the editor June 13, 2006 and, in revised form, August 15, 2006.

2000 Mathematics Subject Classification. Primary 11R06; Secondary 11A67.

Key words and phrases. Univoque, Pisot number, beta-expansion.

Research of the first author was partially supported by MENESR, ACI NIM 154 Numération.

Research of the third author was supported, in part, by NSERC of Canada. 
The last number is the smallest limit point of the set of univoque Pisot numbers (Theorem 5.3). We also prove that 2 is a limit point of univoque Pisot numbers.

\section{DEFInitions AND REMINDERS}

2.1. Infinite words. Let $\mathbb{N}_{+}$denote the set of positive integers. Let $A$ be a finite alphabet. We define $A^{\mathbb{N}_{+}}$to be the set of infinite sequences (or infinite words) on $A$ :

$$
A^{\mathbb{N}_{+}}:=\left\{s=\left(s_{n}\right)_{n \geq 1} \mid \forall n \geq 1, s_{n} \in A\right\} .
$$

This set is equipped with the distance $\rho$ defined by: if $s=\left(s_{n}\right)_{n \geq 1}$ and $v=\left(v_{n}\right)_{n \geq 1}$ belong to $A^{\mathbb{N}_{+}}$, then $\rho(s, v):=2^{-r}$ if $s \neq v$ and $r:=\min \left\{n \mid s_{n} \neq v_{n}\right\}$, and $\rho(s, v)=0$ if $s=v$. The topology on the set $A^{\mathbb{N}_{+}}$is then the product topology, and it makes $A^{\mathbb{N}+}$ a compact metric space.

A sequence $\left(s_{n}\right)_{n \geq 1}$ in $A^{\mathbb{N}_{+}}$is said to be periodic if there exists an integer $T \geq 1$, called a period of the sequence, such that $s_{n+T}=s_{n}$ for all $n \geq 1$. A sequence $\left(s_{n}\right)_{n \geq 1}$ in $A^{\mathbb{N}_{+}}$is said to be eventually periodic if there exists an integer $n_{0} \geq 0$ such that the sequence $\left(s_{n+n_{0}}\right)_{n \geq 1}$ is periodic.

If $w$ is a (finite) word, we denote by $w^{\infty}$ the infinite word obtained by concatenating infinitely many copies of $w$ (this is in particular a periodic sequence, and the length of $w$, usually denoted by $|w|$, is a period).

2.2. Base $\beta$ representations. Let $\beta$ be a real number $>1$. A $\beta$-representation of the real number $x \in[0,1]$ is an infinite sequence of integers $\left(x_{n}\right)_{n \geq 1}$ such that $x=\sum_{n \geq 1} x_{n} \beta^{-n}$. If a representation ends in infinitely many zeros, say, and is of the form $w 0^{\infty}$, then the ending zeros are omitted and the representation is said to be finite. The reader is referred to [24, Chapter 7] for more on these topics.

2.2.1. Greedy representations. A special representation of a number $x$, called the greedy $\beta$-expansion, is the infinite sequence $\left(x_{n}\right)_{n \geq 1}$ obtained by using the greedy algorithm of Rényi 27.

Denote by $\lfloor y\rfloor$ and $\{y\}$ the integer part and the fractional part of the real number $y$. Set $r_{0}:=x$ and, for $n \geq 1$, let $x_{n}:=\left\lfloor\beta r_{n-1}\right\rfloor, r_{n}:=\left\{\beta r_{n-1}\right\}$. Then $x=$ $\sum_{n \geq 1} x_{n} \beta^{-n}$.

Intuitively, the digit $x_{n}$ is chosen so that it is the maximal choice allowed at each step. The digits $x_{n}$ obtained by the greedy algorithm belong to the alphabet $A_{\beta}=\{0,1, \ldots,\lfloor\beta\rfloor\}$ if $\beta$ is not an integer, which will always be the case in this work. It is clear from the definition that amongst the $\beta$-representations of a number, the greedy $\beta$-expansion is the largest in lexicographic order (denoted by $\leq_{l e x}$ and $\left.<_{\text {lex }}\right)$. The greedy $\beta$-expansion of $x$ will be denoted by $d_{\beta}(x):=\left(x_{n}\right)_{n \geq 1}$.

The greedy $\beta$-expansion of 1 plays an important role. Set $d_{\beta}(1)=\left(e_{n}\right)_{n \geq 1}$ and define

$$
d_{\beta}^{*}(1):= \begin{cases}d_{\beta}(1) & \text { if } d_{\beta}(1) \text { is infinite } \\ \left(e_{1} \cdots e_{m-1}\left(e_{m}-1\right)\right)^{\infty} & \text { if } d_{\beta}(1)=e_{1} \cdots e_{m-1} e_{m} \text { is finite. }\end{cases}
$$

Of course if $d_{\beta}(1)$ is finite, the sequence $d_{\beta}^{*}(1)$ is also a $\beta$-representation of 1 .

Denote by $\sigma$ the shift on $A_{\beta}^{\mathbb{N}_{+}}$: for any sequence $s=\left(s_{n}\right)_{n \geq 1}$ in $A_{\beta}^{\mathbb{N}_{+}}$, the sequence $v=\sigma(s)$ is defined by $v=\left(v_{n}\right)_{n \geq 1}:=\left(s_{n+1}\right)_{n \geq 1}$. We recall some useful results. 
Theorem $2.1([26])$. Let $s=\left(s_{n}\right)_{n \geq 1}$ be a sequence in $A_{\beta}^{\mathbb{N}_{+}}$. Then

- the sequence $s$ is the greedy $\beta$-expansion of some $x \in[0,1)$ if and only if

$$
\forall k \geq 0, \quad \sigma^{k}(s)<_{\text {lex }} d_{\beta}^{*}(1),
$$

- the sequence $s$ is the greedy $\beta$-expansion of 1 for some $\beta>1$ if and only if

$$
\forall k \geq 1, \quad \sigma^{k}(s)<_{\text {lex }} s .
$$

2.2.2. Lazy representations. Another distinguished $\beta$-representation of the real number $x$ is the so-called lazy expansion, which is the smallest in the lexicographic order among the $\beta$-representations of $x$ on the alphabet $A_{\beta}$. Denote by $\ell_{\beta}(x)=\left(x_{n}\right)_{n \geq 1}$ the lazy $\beta$-expansion of $x$.

To compute it, intuitively we have to choose $x_{n}$ to be as small as possible at each step. The algorithm to obtain the lazy expansion is the following. Let $B:=$ $\sum_{n \geq 1} \frac{\lfloor\beta\rfloor}{\beta^{n}}=\frac{\lfloor\beta\rfloor}{\beta-1}$. Set $r_{0}:=x$ and, for $n \geq 1$, let $x_{n}:=\max \left(0,\left\lceil\beta r_{n-1}-B\right\rceil\right)$, $r_{n}:=\beta r_{n-1}-x_{n}$. Then $x=\sum_{n \geq 1} x_{n} \beta^{-n}$, where the $\left(x_{n}\right)$ form the lazy $\beta$ expansion.

Let $s=\left(s_{n}\right)_{n \geq 1}$ be in $A_{\beta}^{\mathbb{N}_{+}}$. Denote by $\overline{s_{n}}:=\lfloor\beta\rfloor-s_{n}$ the "complement" of $s_{n}$, and by extension $\bar{s}:=\left(\overline{s_{n}}\right)_{n \geq 1}$. Then the following characterization of lazy expansions holds true.

Theorem $2.2([20,16])$. Let $s=\left(s_{n}\right)_{n \geq 1}$ be a sequence in $A_{\beta}^{\mathbb{N}_{+}}$. Then

- the sequence $s$ is the lazy $\beta$-expansion of some $x \in[0,1)$ if and only if

$$
\forall k \geq 0, \quad \sigma^{k}(\bar{s})<_{\text {lex }} d_{\beta}^{*}(1),
$$

- the sequence $s$ is the lazy $\beta$-expansion of 1 for some $\beta>1$ if and only if

$$
\forall k \geq 1, \quad \sigma^{k}(\bar{s})<_{\text {lex }} s .
$$

Example 2.1. Take $\psi_{1}=\frac{1+\sqrt{5}}{2}$ as the golden ratio. The greedy $\beta$-expansion of 1 is $d_{\psi_{1}}(1)=11, d_{\psi_{1}}^{*}(1)=(10)^{2}$, and the lazy expansion of 1 is $\ell_{\psi_{1}}(1)=01^{\infty}$.

2.3. Univoque real numbers. Following [22, 23, a number $\beta>1$ is said to be univoque if there exists a unique sequence of integers $\left(s_{n}\right)_{n \geq 1}$, with $0 \leq s_{n}<\beta$, such that $1=\sum_{n>1} s_{n} \beta^{-n}$. In this case the sequence $\left(s_{n}\right)_{n \geq 1}$ coincides both with the greedy and with the lazy $\beta$-expansion of 1 . Remark that the number 2 is univoque, but we will be concerned with non-integer real numbers in this paper.

Note that some authors call "univoque" the real numbers $x$ having a unique $\beta$ representation (see [18]). Binary sequences $\left(s_{n}\right)_{n \geq 1}$ such that the convergent sum $\sum_{n \geq 1} s_{n} \beta^{-n}$ uniquely determines the sequence $\left(s_{n}\right)_{n \geq 1}$ are also called "univoque" (see [17]). Nevertheless, for simplicity we keep our notion of "univoque".

Definition 2.1. We define two sets of binary sequences as follows:

- A sequence $s=\left(s_{n}\right)_{n \geq 1}$ in $\{0,1\}^{\mathbb{N}_{+}}$is called self-bracketed if for every $k \geq 1$

$$
\bar{s} \leq_{\text {lex }} \sigma^{k}(s) \leq_{\text {lex }} s .
$$

The set of self-bracketed sequences in $\{0,1\}^{\mathbb{N}_{+}}$is denoted by $\Gamma$.

- If all the inequalities above are strict, the sequence $s$ is said to be strictly self-bracketed. If one of the inequalities is an equality, then $s$ is said to be periodic self-bracketed.

The subset of $\Gamma$ consisting of strictly self-bracketed sequences is denoted by $\Gamma_{\text {strict }}$. 
Remark 2.1. The reader will have noted that the expression "periodic self-bracketed" comes from the fact that $\sigma^{k}(s)=s$ or $\sigma^{k}(s)=\bar{s}$ for some $k \geq 1$ implies that the sequence $s$ is periodic.

With this terminology we can rephrase the following result from [20].

Theorem $2.3(20])$. A sequence in $\{0,1\}^{\mathbb{N}_{+}}$is the unique $\beta$-expansion of 1 for a univoque number $\beta$ in $(1,2)$ if and only if it strictly self-bracketed.

Corollary 2.1. Let $s=\left(s_{n}\right)_{n>1}$ be a sequence in $\{0,1\}^{\mathbb{N}_{+}}$. Suppose that the largest string of consecutive 1 's in $s$ has length $k$, and the largest string of consecutive 0 's has length $n$ (here $k$ and $n$ may be $\infty$.) If $n>k$, then $s$ is not self-bracketed.

There exists a smallest univoque real number in $(1,2), 22$. Recall first that the Thue-Morse sequence is the fixed point beginning with 0 of the morphism $0 \rightarrow 01$, $1 \rightarrow 10$ (see for example [5]), hence the sequence

$$
0110100110010110 \ldots
$$

Theorem $2.4([22])$. There exists a smallest univoque real number $\kappa \in(1,2)$. One has $\kappa \approx 1.787231$, and $d_{\kappa}(1)=\left(t_{n}\right)_{n \geq 1}$, where $\left(t_{n}\right)_{n \geq 1}=11010011 \ldots$ is obtained by shifting the Thue-Morse sequence.

The number $\kappa$ is not rational; actually more can be proved.

Theorem 2.5 (3]). The Komornik-Loreti constant $\kappa$ is transcendental.

Notation. In the remainder of this paper, we will denote by $\mathcal{U}$ the set of real numbers in $(1,2)$ which are univoque. We will denote by $\widetilde{\mathcal{U}}$ the set of real numbers $\beta \in(1,2)$ such that $d_{\beta}(1)$ is finite and $d_{\beta}^{*}(1)$ is a periodic self-bracketed sequence.

Formally, we have

$$
\mathcal{U}=\left\{\beta \in(1,2): d_{\beta}(1) \in \Gamma_{\text {strict }}\right\}
$$

and

$$
\widetilde{\mathcal{U}}=\left\{\beta \in(1,2): d_{\beta}(1) \text { is finite and } d_{\beta}^{*}(1) \text { is periodic self-bracketed }\right\} .
$$

2.4. Pisot numbers. A Pisot number is an algebraic integer $>1$ such that all its algebraic conjugates (other than itself) have modulus $<1$. As usual the set of Pisot numbers is denoted by $S$ and its derived set (set of limit points) by $S^{\prime}$. It is known that $S$ is closed [28, and has a smallest element, which is the root $>1$ of the polynomial $x^{3}-x-1$ (approx. 1.3247). A Salem number is an algebraic integer $>1$ such that all its algebraic conjugates have modulus $\leq 1$, with at least one conjugate on the unit circle.

We recall some results on Pisot and Salem numbers (the reader is referred to 7 for more on these topics). One important result is that if $\beta$ is a Pisot number, then $d_{\beta}(1)$ is eventually periodic (finite or infinite) 8 . Note that $d_{\beta}(1)$ is never periodic, but that when $d_{\beta}(1)$ is finite, $d_{\beta}^{*}(1)$ is periodic. A number $\beta$ such that $d_{\beta}(1)$ is eventually periodic is called a Parry number (they are called beta-numbers by Parry [26]). When $d_{\beta}(1)$ is finite, $\beta$ is called a simple Parry number.

One deeper result is the following one.

Theorem 2.6 ([8, 29]). Let $\beta$ be a Pisot number. A number $x$ of $[0,1]$ has a (finite or infinite) eventually periodic greedy $\beta$-expansion if and only if it belongs to $\mathbb{Q}(\beta)$.

For lazy expansions we have a similar result. 
Corollary 2.2. Let $\beta$ be a Pisot number. A number $x$ of $[0,1]$ has an eventually periodic lazy $\beta$-expansion if and only if it belongs to $\mathbb{Q}(\beta)$.

Proof. Let $\ell_{\beta}(x)=\left(x_{n}\right)_{n \geq 1}$. By Theorem 2.2 the sequence $\left(\overline{x_{n}}\right)_{n \geq 1}$ is the greedy $\beta$-expansion of the number $\frac{\lfloor\beta\rfloor}{\beta-1}-x$, and the result follows from Theorem 2.6 .

Amara has determined all the limit points of $S$ smaller than 2 in 6 .

Theorem 2.7 ([6]). The limit points of $S$ in $(1,2)$ are the following:

$$
\varphi_{1}=\psi_{1}<\varphi_{2}<\psi_{2}<\varphi_{3}<\chi<\psi_{3}<\varphi_{4}<\cdots<\psi_{r}<\varphi_{r+1}<\cdots<2
$$

where

$$
\left\{\begin{array}{l}
\text { the minimal polynomial of } \varphi_{r} \text { is } x^{r+1}-2 x^{r}+x-1 \\
\text { the minimal polynomial of } \psi_{r} \text { is } x^{r+1}-x^{r}-\cdots-x-1 \\
\text { the minimal polynomial of } \chi \text { is } x^{4}-x^{3}-2 x^{2}+1
\end{array}\right.
$$

The first few limit points are:

- $\varphi_{1}=\psi_{1} \approx 1.618033989$, the root in $(1,2)$ of $x^{2}-x-1$,

- $\varphi_{2} \approx 1.754877666$, the root in $(1,2)$ of $x^{3}-2 x^{2}+x-1$,

- $\psi_{2} \approx 1.839286755$, the root in $(1,2)$ of $x^{3}-x^{2}-x-1$,

- $\varphi_{3} \approx 1.866760399$, the root in $(1,2)$ of $x^{4}-2 x^{3}+x-1$,

- $\chi \approx 1.905166168$, the root in $(1,2)$ of $x^{4}-x^{3}-2 x^{2}+1$,

- $\psi_{3} \approx 1.927561975$, the root in $(1,2)$ of $x^{4}-x^{3}-x^{2}-x-1$.

The greedy and lazy $\beta$-expansions of these points are given in Table 1 below.

For any interval $[a, b]$, with $b<2$, an algorithm of Boyd 10, 11, 12 finds all Pisot numbers in the interval. If $[a, b]$ contains a limit point $\theta$, then there exists an $\varepsilon>0$ such that all Pisot numbers in $[\theta-\varepsilon, \theta+\varepsilon]$ are regular Pisot numbers of a known form. Boyd's algorithm detects these regular Pisot numbers, and truncates the search accordingly. (For a non-effective study of Pisot numbers in subintervals of $(1,2)$, see also 30,31 .)

Recall that Boyd has shown that for any Salem number of degree 4 the greedy expansion of 1 is eventually periodic, [13, and has given some evidence in favor of the conjecture that it is still the case for degree $6,[15]$.

\section{Preliminary combinatorial Results}

We start by defining a function $\Phi$ on the infinite words of the form $b=(z 0)^{\infty}$.

Definition 3.1. Let $b=(z 0)^{\infty}$ be a periodic binary word whose period pattern ends in a 0 . Suppose furthermore that the minimal period of $b$ is equal to $1+|z|$. Then we define $\Phi(b)$ by

$$
\Phi(b):=(z 1 \bar{z} 0)^{\infty} .
$$

We now recall a result from [1].

\section{Lemma 3.1.}

- If a sequence $b$ belonging to $\Gamma$ begins with un where $u$ is a finite non-empty word, then $b=(u \bar{u})^{\infty}$.

- If $b=(z 0)^{\infty}$, where the minimal period of $b$ is equal to $1+|z|$, is an element of $\Gamma$, then $\Phi(b)$ belongs to $\Gamma$, and there is no element of $\Gamma$ lexicographically between $b$ and $\Phi(b)$. 
Remark 3.1. The inequalities defining the set $\Gamma$ show that $\Gamma$ is a (topologically) closed set.

Corollary 3.1. Let $b=(z 0)^{\infty}$ (where the minimal period of $b$ is equal to $1+|z|$ ). The sequence $\left(\Phi^{(n)}(b)\right)_{n \geq 0}$ is a sequence of elements of $\Gamma$ that converges to a limit $\Phi^{(\infty)}(b)$ in $\Gamma$. The only elements of $\Gamma$ lexicographically between $b$ and $\Phi^{(\infty)}(b)$ are the $\Phi^{(k)}(b), k \geq 0$.

By abuse of notation, if $\theta$ is the number such that $d_{\theta}^{*}(1)=b$, we denote by $\Phi(\theta)$ the real number $>1$ such that $d_{\Phi(\theta)}^{*}(1)=\Phi(b)$.

Take $b=d_{\psi_{r}}^{*}(1)=\left(1^{r} 0\right)^{\infty}$. Then $\Phi(b)=\left(1^{r} 10^{r} 0\right)^{\infty}=d_{\varphi_{r+1}}^{*}(1)$, thus $\varphi_{r+1}=$ $\Phi\left(\psi_{r}\right)$. Let $\pi_{r}$ be the real number defined by $d_{\pi_{r}}^{*}(1)=\Phi^{(\infty)}\left(\left(1^{r} 0\right)^{\infty}\right)$, that is, $\pi_{r}=\Phi^{\infty}\left(\psi_{r}\right)$. Then $d_{\pi_{r}}^{*}(1)$ is strictly self-bracketed (see [1]), hence the following result holds true.

Proposition 3.1. The number $\pi_{r}$ is univoque. Furthermore between $\psi_{r}$ and $\pi_{r}=$ $\Phi^{(\infty)}\left(\psi_{r}\right)$ the only real numbers belonging to $\mathcal{U}$ or $\widetilde{\mathcal{U}}$ are the numbers $\varphi_{r+1}, \Phi\left(\varphi_{r+1}\right)$, $\Phi^{(2)}\left(\varphi_{r+1}\right)$, etc. They all belong to $\widetilde{\mathcal{U}}$.

We will now prove a combinatorial property of the sequences $d_{\beta}(1)$. Before stating and proving this property we first make a straightforward remark.

Remark 3.2. Let $u$ and $v$ be two binary words having the same length. Let $a$ and $b$ be either two binary words having the same length or two infinite binary sequences. Suppose that $a$ begins with $u$ and $b$ begins with $v$. Then

$$
\begin{aligned}
& a \leq_{\text {lex }} b \quad \Longrightarrow \quad u \leq_{\text {lex }} v, \\
& u<_{\text {lex }} v \Longrightarrow a<_{\text {lex }} b .
\end{aligned}
$$

Proposition 3.2. Let $a=(w 0)^{\infty}$ be an infinite periodic binary sequence with minimal period $1+|w|$, such that $w$ (and hence a) begins in 1 . Let $b=w 10^{\infty}$. Then the following two properties are equivalent:

(i) $\forall k \geq 1, \quad \sigma^{k}(a) \leq_{\text {lex }} a$,

(ii) $\forall k \geq 1, \sigma^{k}(b)<_{\text {lex }} b$.

Proof. We first prove (i) $\Longrightarrow$ (ii). Since we clearly have $\sigma^{k}(b)<_{\text {lex }} b$ for each $k \geq|w|$, we can suppose that $k<|w|$. Write $w=u v$ where $|u|=k$, hence $u$ and $v$ are both non-empty. This gives $a=(u v 0)^{\infty}$ and $b=u v 10^{\infty}$, and we want to prove that $v 10^{\infty}<_{\text {lex }} u v 10^{\infty}$.

Let us write $|v|=d|u|+e$, where $d \geq 0$ and $e \in[0,|u|)$. We can write $v=$ $v_{1} v_{2} \ldots v_{d} z$, with $\left|v_{1}\right|=\left|v_{2}\right|=\ldots=\left|v_{d}\right|=|u|$, and $|z|=e<|u|$. Note that, if $d=0$, then $v=z$.

Let us also write $u=s t$ and, for each $j \in[1, d], v_{j}=s_{j} t_{j}$, where $|s|=\left|s_{1}\right|=$ $\left|s_{2}\right|=\ldots=\left|s_{d}\right|=|z|$ and $|t|=\left|t_{1}\right|=\left|t_{2}\right|=\ldots=\left|t_{d}\right|$. We thus have

$$
a=\left(s t s_{1} t_{1} s_{2} t_{2} \ldots s_{d} t_{d} z 0\right)^{\infty}
$$

and we want to prove that

$$
s_{1} t_{1} s_{2} t_{2} \ldots s_{d} t_{d} z 10^{\infty}<_{\text {lex }} s t s_{1} t_{1} s_{2} t_{2} \ldots s_{d} t_{d} z 10^{\infty} .
$$

Applying, for each $j \in[1, d]$, the hypothesis $\sigma^{k}(a) \leq_{l e x} a$ with $k=\mid s t s_{1} t_{1} s_{2} t_{2} \ldots$ $s_{j-1} t_{j-1} \mid$ (in particular if $j=1$, then $k=|s t|$ ), we see that $s_{j} t_{j} \leq_{\text {lex }} s t$. Define

$$
\mathcal{E}:=\left\{j, s_{j} t_{j}<_{\text {lex }} s t\right\} .
$$


- If $\mathcal{E} \neq \emptyset$, let $j_{0}=\min \mathcal{E}$. Then

$$
s t=s_{1} t_{1}=s_{2} t_{2}=\ldots=s_{j_{0}-1} t_{j_{0}-1},
$$

i.e.,

$$
s=s_{1}=s_{2}=\ldots=s_{j_{0}-1} \text { and } t=t_{1}=t_{2}=\ldots=t_{j_{0}-1}
$$

(this condition is empty if $j_{0}=1$ ) and

$$
s_{j_{0}} t_{j_{0}}<_{\text {lex }} s t \text {. }
$$

In this case we have $b=(s t)^{j_{0}} s_{j_{0}} t_{j_{0}} \ldots s_{d} t_{d} z 10^{\infty}$, and we want to prove that

$$
(s t)^{j_{0}-1} s_{j_{0}} t_{j_{0}} \ldots s_{d} t_{d} z 10^{\infty}<_{l e x}(s t)^{j_{0}} s_{j_{0}} t_{j_{0}} \ldots s_{d} t_{d} z 10^{\infty}
$$

which is an immediate consequence of the inequality $s_{j_{0}} t_{j_{0}}<_{l e x} s t$.

- If $\mathcal{E}=\emptyset$, then either $d=0$, or $s_{1} t_{1}=s_{2} t_{2}=\ldots=s_{d} t_{d}=s t$. Either way, we get

$$
s_{1}=s_{2}=\ldots=s_{d}=s \text { and } t_{1}=t_{2}=\ldots=t_{d}=t .
$$

In this case we have $a=\left((s t)^{d+1} z 0\right)^{\infty}$ and we want to prove that $(s t)^{d} z 10^{\infty}$ $<_{\text {lex }}(s t)^{d+1} z 10^{\infty}$, i.e., that $z 10^{\infty}<_{\text {lex }}$ st $z 10^{\infty}$. Applying the hypothesis $\sigma^{k}(a) \leq_{l e x} a$ with $k=\left|(s t)^{d+1}\right|$, we see that $z \leq_{l e x} s$.

- If $z<_{\text {lex }} s$, the inequality $z 10^{\infty}<_{\text {lex }} s t z 10^{\infty}$ is clear.

- If $z=s$, we want to prove that $10^{\infty}<_{\text {lex }} t z 10^{\infty}$, i.e., that $t$ begins in 1 (note that, if $t$ is empty, then the inequality is clear since $z=s$ begins in 1 as does $a$ ). If we had $t=0 r$, with $r$ possibly empty, we would have $a=\left((z 0 r)^{d+1} z 0\right)^{\infty}$. Applying the hypothesis $\sigma^{k}(a) \leq_{\text {lex }} a$ with $k=\left|(z 0 r)^{d+1}\right|$ and $k=\left|(z 0 r)^{d} z 0\right|$ we get respectively $z 0 z 0 r \leq_{\text {lex }} z 0 r z 0$ (i.e., $\left.z 0 r \leq_{l e x} r z 0\right)$ and $r z 0 \leq_{l e x} z 0 r$. Hence we have $r z 0=z 0 r$.

Writing this last equality as $r(z 0)=(z 0) r$, the Lyndon-Schützenberger theorem (see [25]) implies that $r=\emptyset$ or there exist a non-empty word $x$ and two integers $p, q \geq 1$, such that $z 0=x^{p}$ and $r=x^{q}$. This gives $a=\left(x^{a(d+2)}\right)^{\infty}$ or $a=\left(x^{(p+q)(d+1)+p}\right)^{\infty}$. In both cases $a=x^{\infty}$ and $|x|<\left|\left((z 0 r)^{d+1} z 0\right)\right|$ which contradicts the minimality of the period of $a$.

We now prove (ii) $\Longrightarrow$ (i). Because of the periodicity of the sequence $a$ and the fact that it begins in 1 , we can suppose that $k \leq|w|$. Hence we write $w=u v$ with $u$ and $v$ nonempty and $|u|=k$, and we want to prove that $v 0(u v 0)^{\infty} \leq_{l e x}(u v 0)^{\infty}$. Since $u$ begins in 1 as $a$ does, it suffices to prove that $v 01^{\infty} \leq_{l e x}(u v 0)^{\infty}$. Applying the hypothesis $\sigma^{k}(b)<_{\text {lex }} b$ with $k=|u|$, we have $v 10^{\infty}<_{\text {lex }} u v 10^{\infty}$.

Hence $v 10^{|u|} \leq_{l e x} u v 1$. This inequality must be strict since its left-hand side ends in a 0 and its right-hand side ends with a 1 : thus $v 10^{|u|}<_{l e x} u v 1$. Hence $v 10^{|u|} \leq_{l e x} u v 0$.

We then can write $v 0 u<_{\text {lex }} v 10^{|u|} \leq_{\text {lex }} u v 0$, hence $v 0 u<_{\text {lex }} u v 0$. This implies in turn $v 0(u v 0)^{\infty} \leq_{l e x}(u v 0)^{\infty}$.

Corollary 3.2. The sequence $a=(w 0)^{\infty}$ is equal to $d_{\theta}^{*}(1)$ for some $\theta>1$ if and only if $b=w 10^{\infty}$ is equal to $d_{\theta}(1)$.

We end this section with a result on limits of sequences of elements in $\Gamma$. 
Lemma 3.2. A sequence of $\Gamma$ of the form $(w 0)^{\infty}$ cannot be a limit from above of a non-eventually constant sequence of elements of $\Gamma$.

Proof. Suppose we have a sequence $\left(z^{(m)}\right)_{m \geq 0}$ with $z^{(m)}=\left(z_{n}^{(m)}\right)_{n \geq 1}$ belonging to $\Gamma$, and converging towards $(w 0)^{\infty}$, with $z^{(m)} \geq(w 0)^{\infty}$. From Lemma 3.1 there is no element of $\Gamma$ lexicographically between $(w 0)^{\infty}$ and $(w 1 \bar{w} 0)^{\infty}$, hence $\left(z^{(m)}\right)_{m \geq 0}$ is ultimately equal to $(w 0)^{\infty}$.

\section{First RESUlts}

In this section we consider only numbers $\beta$ belonging to $(1,2)$.

4.1. Preliminary results. Our goal here is to present some simple preliminary data. In particular, in Table 1, we give the expansions for Pisot numbers in $S^{\prime} \cap$ $(1,2)$, in Table 2 we give Pisot numbers of small degree in the interval $(1,2)$, and in Table 3 we examine Salem numbers of small degree in the interval $(1,2)$. Some observations that are worth making, based on these tables, include:

Remark 4.1.

- The golden ratio $\varphi_{1}=\psi_{1}$ is the smallest element of $\tilde{\mathcal{U}}$. (This comes straight from Definition 2.1.)

- There is no univoque Pisot number of degree 2 or 3.

- The number $\chi$ is the unique Pisot number of degree 4 which is univoque.

- For Pisot numbers $\psi_{r}$, the lazy expansion coincides with $d_{\psi_{r}}^{*}(1)$.

- There exists a unique Salem number of degree 4 which is univoque.

- Salem numbers greater than the Komornik-Loreti constant $\kappa$ appear to be univoque (for degrees 4 and 6).

TABLE 1. Greedy and lazy $\beta$-expansions of real numbers in $S^{\prime} \cap(1,2)$.

\begin{tabular}{|lllll|}
\hline Minimal & $\begin{array}{l}\text { Pisot } \\
\text { Polynomial }\end{array}$ & $\begin{array}{l}\text { Greedy } \\
\text { Number }\end{array}$ & $\begin{array}{l}\text { Lazy } \\
\text { expansion }\end{array}$ & Comment \\
\hline$x^{r+1}-2 x^{r}+x-1$ & $\varphi_{r}$ & $1^{r} 0^{r-1} 1$ & $1^{r-1} 01^{\infty}$ & periodic self-bracketed \\
$x^{r+1}-x^{r}-\cdots-1$ & $\psi_{r}$ & $1^{r+1}$ & $\left(1^{r} 0\right)^{\infty}$ & periodic self-bracketed \\
$x^{4}-x^{3}-2 x^{2}+1$ & $\chi$ & $11(10)^{\infty}$ & $11(10)^{\infty}$ & univoque \\
\hline
\end{tabular}

We also observe the following lemma which is straightforward.

Lemma 4.1. A Parry number which is univoque must be a unit (i.e., an algebraic integer whose minimal polynomial has its constant term equal to \pm 1 ).

For each Pisot or Salem number of degree less than 4 or 6 respectively, we simply compute the greedy and lazy expansion, and then compare them to see when they are equal. To find the list of Pisot numbers, we use the algorithm of Boyd [10]. Although there is no nice algorithm to find Salem numbers in $(1,2)$ of fixed degree, for low degree we can use brute force. Namely, if $P(x)=x^{n}+a_{1} x^{n-1}+\cdots+a_{1} x+1$ is a Salem polynomial with root in $(1,2)$ and $Q(x)=x^{n}+b_{1} x^{n-1}+\cdots+b_{1} x+1=$ $(x+2)(x+1 / 2)(x+1)^{n-2}$, then we have $\left|a_{i}\right| \leq b_{i}$. See [9] for more on bounds of coefficients. 
TABLe 2. Greedy and lazy expansions of degree 2, 3 and 4 Pisot numbers.

\begin{tabular}{|lllll|}
\hline Minimal polynomial & Pisot number & $\begin{array}{l}\text { Greedy } \\
\text { expansion }\end{array}$ & $\begin{array}{l}\text { Lazy } \\
\text { expansion }\end{array}$ & Comment \\
\hline$x^{2}-x-1$ & 1.618033989 & 11 & $01^{\infty}$ & periodic self-bracketed \\
& & & & \\
$x^{3}-x-1$ & 1.324717957 & 10001 & $00001^{\infty}$ & \\
$x^{3}-x^{2}-1$ & 1.465571232 & 101 & $001^{\infty}$ & \\
$x^{3}-2 x^{2}+x-1$ & 1.754877666 & 1101 & $101^{\infty}$ & periodic self-bracketed \\
$x^{3}-x^{2}-x-1$ & 1.839286755 & 111 & $(110)^{\infty}$ & periodic self-bracketed \\
& & & & \\
$x^{4}-x^{3}-1$ & 1.380277569 & 1001 & $0001^{\infty}$ & \\
$x^{4}-2 x^{3}+x-1$ & 1.866760399 & 111001 & $1101^{\infty}$ & periodic self-bracketed \\
$x^{4}-x^{3}-2 x^{2}+1$ & 1.905166168 & $11(10)^{\infty}$ & $11(10)^{\infty}$ & univoque \\
$x^{4}-x^{3}-x^{2}-x-1$ & 1.927561975 & 1111 & $(1110)^{\infty}$ & periodic self-bracketed \\
\hline
\end{tabular}

TABLe 3. Greedy and lazy expansions of degree 4 and 6 Salem numbers. Here $w=011010110$.

\begin{tabular}{|c|c|c|c|c|}
\hline Minimal polynomial & $\begin{array}{l}\text { Salem } \\
\text { number }\end{array}$ & $\begin{array}{l}\text { Greedy } \\
\text { expansion }\end{array}$ & $\begin{array}{l}\text { Lazy } \\
\text { expansion }\end{array}$ & Comment \\
\hline$x^{4}-x^{3}-x^{2}-x+1$ & 1.722083806 & $1(100)^{\infty}$ & $101(110)^{\infty}$ & \\
\hline$x^{4}-2 x^{3}+x^{2}-2 x+1$ & 1.883203506 & $1(1100)^{\infty}$ & $1(1100)^{\infty}$ & univoque \\
\hline$x^{6}-x^{4}-x^{3}-x^{2}+1$ & 1.401268368 & $1(0010000)^{\infty}$ & $0010111(1111110)^{\infty}$ & \\
\hline$x^{6}-x^{5}-x^{3}-x+1$ & 1.506135680 & $1(01000)^{\infty}$ & $01011(11110)^{\infty}$ & \\
\hline$x^{6}-x^{5}-x^{4}+x^{3}-x^{2}-x+1$ & 1.556030191 & $1(01001001000)^{\infty}$ & $01^{3}(01)^{2}\left(1^{7} 01^{6} w 1^{3} w 1^{6}\right.$ & $0)^{\infty}$ \\
\hline$x^{6}-x^{4}-2 x^{3}-x^{2}+1$ & 1.582347184 & $1(0101000)^{\infty}$ & $011(110)^{\infty}$ & \\
\hline $\begin{array}{l}x^{6}-2 x^{5}+2 x^{4}-3 x^{3} \\
\quad+2 x^{2}-2 x+1\end{array}$ & 1.635573130 & $1(1000000100)^{\infty}$ & $1010101(1101111110)^{\infty}$ & \\
\hline$x^{6}-x^{5}-x^{4}-x^{2}-x+1$ & 1.781643599 & $1(10100)^{\infty}$ & $11001(11110)^{\infty}$ & \\
\hline$x^{6}-2 x^{5}+x^{3}-2 x+1$ & 1.831075825 & $1(10110100)^{\infty}$ & $1(10110100)^{\infty}$ & univoque \\
\hline$x^{6}-x^{5}-x^{4}-x^{3}-x^{2}-x+1$ & 1.946856268 & $1(11100)^{\infty}$ & $1(11100)^{\infty}$ & univoque \\
\hline $\begin{aligned} x^{6} & -2 x^{5}-x^{4}+3 x^{3}-x^{2} \\
& -2 x+1\end{aligned}$ & 1.963553039 & $1(111011100)^{\infty}$ & $1(111011100)^{\infty}$ & univoque \\
\hline $\begin{array}{l}x^{6}-2 x^{5}+x^{4}-2 x^{3}+x^{2} \\
\quad-2 x+1\end{array}$ & 1.974818708 & $1(111100)^{\infty}$ & $1(111100)^{\infty}$ & univoque \\
\hline$x^{6}-2 x^{4}-3 x^{3}-2 x^{2}+1$ & 1.987793167 & $1(1111100)^{\infty}$ & $1(1111100)^{\infty}$ & univoque \\
\hline
\end{tabular}

4.2. Limit points of univoque numbers. In this section we concern ourselves with the structure of $\mathcal{U} \cap S$ and $\widetilde{\mathcal{U}} \cap S$, as well as intersections with the derived set $S^{\prime}$. We begin with the following result.

Proposition 4.1. The limit of a sequence of real numbers belonging to $\mathcal{U}$ belongs to $\mathcal{U}$ or $\tilde{\mathcal{U}}$.

Proof. Let $\left(\theta_{j}\right)_{j \geq 1}$ be a sequence of numbers belonging to $\mathcal{U}$ such that $\lim _{j \rightarrow \infty} \theta_{j}=$ $\theta$. Let $a^{(j)}=\left(a_{n}^{(j)}\right)_{n \geq 1}:=d_{\theta_{j}}(1)$. Up to replacing the sequence $\left(\theta_{j}\right)_{j \geq 1}$ by a subsequence, we may assume that the sequence of sequences $\left(a_{n}^{(j)}\right)_{n \geq 1}$ converges to a limit $a=\left(a_{n}\right)_{n \geq 1}$ when $j$ goes to infinity. Then (dominated convergence):

$$
1=\sum_{n \geq 1} \frac{a_{n}}{\theta^{n}}
$$

For every $j \geq 1$ the number $\theta_{j}$ belongs to $\mathcal{U}$. Hence the sequence $a^{(j)}$ belongs to $\Gamma_{\text {strict }}$, hence to $\Gamma$. Thus the $\operatorname{limit}_{a} a=\lim _{j \rightarrow \infty} a^{(j)}$ belongs to $\Gamma$ (see Remark 3.1).

If $a$ belongs to $\Gamma_{\text {strict }}$, then it is the $\theta$-expansion of 1 , and $\theta$ belongs to $\mathcal{U}$. 
If $a$ is periodic self-bracketed, it is of the form $a=(w 0)^{\infty}$, where we may assume that the minimal period of $a$ is $1+|w|$. From Corollary [3.2, $a=d_{\theta}^{*}(1)$, $b:=w 10^{\infty}=d_{\theta}(1)$, and $\theta$ belongs to the set $\widetilde{\mathcal{U}}$.

Corollary 4.1. The numbers $\varphi_{r}$ cannot be limit points of numbers in $\mathcal{U}$.

Proof. This is a consequence of the first part of Lemma 3.1

We now give two remarkable sequences of real numbers that converge to the Komornik-Loreti constant $\kappa$. Part (ii) of Proposition 4.2 below was obtained independently by the second author and in 23 .

\section{Proposition 4.2 .}

(i) Let $t=\left(t_{n}\right)_{n \geq 1}=11010011 \ldots$ be the shifted Thue-Morse sequence, and let $\tau_{2^{k}}$ be the real number $>1$ such that $d_{\tau_{2^{k}}}(1)=t_{1} \cdots t_{2^{k}}$. Then, the sequence of real numbers $\left(\tau_{2^{k}}\right)_{k \geq 1}$ converges from below to the KomornikLoreti constant $\kappa$. These numbers belong to $\widetilde{\mathcal{U}}$. The first three are Pisot numbers.

(ii) There exists a sequence of univoque Parry numbers that converges to $\kappa$ from above.

Proof. To prove (i), note that $\tau_{2}$ is the golden ratio, $\tau_{4}=\Phi\left(\tau_{2}\right)=\varphi_{2}, \tau_{8}=\Phi^{2}\left(\tau_{2}\right)$, etc., and $\kappa=\Phi^{(\infty)}\left(\tau_{2}\right)$.

In order to prove (ii) we define $\delta_{2^{k}}$ as the number such that

$$
d_{\delta_{2^{k}}}(1)=t_{1} \cdots t_{2^{k}-1}\left(1 \overline{t_{1}} \cdots \overline{{t^{k}-1}}\right)^{\infty} \text {. }
$$

Clearly the sequence $d_{\delta_{2 k}}$ (1) converges to $t$ when $k$ goes to infinity, and thus the sequence $\left(\delta_{2^{k}}\right)_{k \geq 1}$ converges to $\kappa$.

Remark 4.2.

- Let $Q_{2^{k}}$ be the polynomial "associated" with $\tau_{2^{k}}$ : writing $1=\sum_{1 \leq j \leq 2^{k}} \frac{t_{j}}{\tau_{2^{k}}^{j}}$ immediately gives a polynomial $Q_{2^{k}}(x)$ of degree $2^{k}$ such that $Q_{2^{k}}\left(\tau_{2^{k}}\right)=$ 0 . Then, for $k \geq 2$, the polynomial $Q_{2^{k}}(x)$ is divisible by the product $(x+1)\left(x^{2}+1\right) \cdots\left(x^{2^{k-2}}+1\right)$.

- Let $R_{2^{k}}(x)$ be the polynomial of degree $2^{k+1}-1$ associated (as above) with $\delta_{2^{k}}$. Then it can be shown that, for $k \geq 2$, the polynomial $R_{2^{k}}(x)$ is divisible by the same product $(x+1)\left(x^{2}+1\right) \cdots\left(x^{2^{k-2}}+1\right)$.

\section{MAIN RESUltS}

Recall that Amara gave in [6] a complete description of the limit points of the Pisot numbers in the interval $(1,2)$ (see Theorem 2.7). Talmoudi 31] gave a description for sequences of Pisot numbers approaching each of the values $\varphi_{r}, \psi_{r}$ or $\chi$. The Pisot numbers in these sequences are called regular Pisot numbers. Further, Talmoudi showed that, for all $\varepsilon>0$, there are only a finite number of Pisot numbers in $(1,2-\varepsilon)$, that are not in one of these sequences. These are called the irregular Pisot numbers, and they will be examined later in Section 5.3.

Since $\chi$ is a univoque Pisot number (Tables 1 and 2), it is natural to ask if there are any other univoque Pisot numbers smaller than $\chi$. As well, it is natural to ask if there is a smallest univoque Pisot number. This leads us to our first result: 
Theorem 5.1. There exists a smallest Pisot number in the set $\mathcal{U}$.

Proof. Define $\theta$ by $\theta:=\inf (S \cap \mathcal{U})$. We already know that $\theta$ belongs to $S$, since $S$ is closed. On the other hand, from Proposition 4.1, either $\theta$ belongs to $\mathcal{U}$ or to $\widetilde{\mathcal{U}}$. It suffices to show that $\theta$ cannot belong to $\widetilde{\mathcal{U}}$. If it were the case, first $\theta$ would be a limit point of elements of $(S \cap \mathcal{U})$. On the other hand we could write $d_{\theta}^{*}(1)=(w 0)^{\infty}$, with the minimal period of the sequence $d_{\theta}^{*}(1)$ being $1+|w|$ (note that $\theta<\chi$ since $\chi$ belongs to $(S \cap \mathcal{U})$ and $\theta \neq \chi)$. But from Lemma 3.2 there is a contradiction.

Now, to find the univoque Pisot numbers less than $\chi$, we need to examine the irregular Pisot numbers less than $\chi$ (Section 5.3). We also need to examine the infinite sequences of Pisot numbers tending to those $\varphi_{r}$ and $\psi_{r}$ less than $\chi$. Lastly, we need to examine the sequences of Pisot numbers tending to $\chi$ from below.

By noticing that $\varphi_{1}=\psi_{1}$ and $\varphi_{2}$ are all strictly less than $\kappa$, the Komornik-Loreti constant, we can disregard these limit points. Further, we may disregard $\varphi_{3}$ as a limit point by Corollary 4.1. In particular:

Proposition 5.1. There are no univoque numbers between $\psi_{2}$ and 1.8705 . (Note that $1.8705>\varphi_{3}$.)

Proof. We easily see from Proposition 3.1 that

$$
\Phi^{2}\left(\psi_{2}\right)=\Phi\left(\varphi_{3}\right) \approx 1.870556617
$$

which gives the result.

So we see that it suffices to examine the sequence of Pisot numbers tending towards $\psi_{2}$ from below, and those tending to $\chi$ from below.

5.1. Approaching $\psi_{2}$ from below. We know that the $\psi_{r}$ are limit points of the set of Pisot numbers. Moreover, we know exactly what the sequences tending to $\psi_{r}$ look like. Let $P_{\psi_{r}}(x)=x^{r+1}-\cdots-1$ be the Pisot polynomial associated with $\psi_{r}$. Let $A_{\psi_{r}}(x)=x^{r+1}-1$ and $B_{\psi_{r}}(x)=\frac{x^{r}-1}{x-1}$ be two polynomials associated with $P_{\psi_{r}}(x) 11$ Then for sufficiently large $n$, the polynomials $P_{\psi_{r}}(x) x^{n} \pm A_{\psi_{r}}(x)$ and $P_{\psi_{r}}(x) x^{n} \pm B_{\psi_{r}}(x)$ admit a unique root between 1 and 2, which is a Pisot number. These sequences of Pisot numbers are the regular Pisot numbers associated with $\psi_{r}$. See for example [6, 14].

Moreover, we have that the roots of $P_{\psi_{r}}(x) x^{n}-A_{\psi_{r}}(x)$ and $P_{\psi_{r}}(x) x^{n}-B_{\psi_{r}}(x)$ approach $\psi_{r}$ from above, and those of $P_{\psi_{r}}(x) x^{n}+A_{\psi_{r}}(x)$ and $P_{\psi_{r}}(x) x^{n}+B_{\psi_{r}}(x)$ approach $\psi_{r}$ from below. This follows as $P_{\psi_{r}}(1)=-1$ and $P_{\psi_{r}}(2)=1$, with $P_{\psi_{r}}(x)$ strictly increasing on $[1,2]$, along with the fact that on $(1,2]$ we have $A_{\psi_{r}}(x), B_{\psi_{r}}(x)>0$. Although we need only examine the sequences of Pisot numbers approaching $\psi_{2}$ from below, we give the results for all sequences approaching $\psi_{2}$ for completeness.

Lemma 5.1. The greedy and lazy expansions of Pisot numbers approaching $\psi_{2}$ are summarized in Table 4 .

Remark 5.1. It is interesting to observe that, in the case $P_{\psi_{2}}(x) x^{n}-B_{\psi_{2}(x)}(x)$ (last section of Table (4), for $n=2,3$ and 4 , the lazy expansion $\ell_{\beta}(1)$ is equal to $d_{\beta}^{*}(1)$.

\footnotetext{
${ }^{1}$ Note that the definition of $B_{\psi_{r}}(x)$ is different from the definition in 14, and corrects a misprint in that paper.
} 
TABLE 4. Greedy and lazy expansion for regular Pisot numbers approaching $\psi_{2}$.

\begin{tabular}{|c|c|c|c|}
\hline Case & Greedy expansion & Lazy expansion & Comment \\
\hline \multicolumn{4}{|c|}{$=\quad P_{\psi_{2}}(x) x^{n}+A_{\psi_{2}}(x)$} \\
\hline$n=1$ & 101 & $00(1)^{\infty}$ & \\
\hline$n=2$ & 10101 & $0(11101)^{\infty}$ & \\
\hline$n=3$ & 110001 & $1010(1)^{\infty}$ & \\
\hline$n=4$ & 1100110001 & $10(1111110011)^{\infty}$ & \\
\hline$n=3 k+1$ & $(110)^{k} 011(000)^{k} 1$ & $(110)^{k} 0(101)^{\infty}$ & \\
\hline$n=3 k+2$ & $1(101)^{k} 010(000)^{k} 1$ & $1(101)^{k} 0(011)^{\infty}$ & \\
\hline$n=3 k+3$ & $(110)^{k+1} 00(000)^{k} 1$ & $1(101)^{k} 0\left((110)^{k+1} 01(101)^{k} 1\right)^{\infty}$ & \\
\hline \multicolumn{4}{|c|}{$P_{P_{\psi_{2}}}(x) x^{n}-A_{\psi_{2}}(x)$} \\
\hline$n=1$ & Root bigger than 2 & & \multirow{7}{*}{$\begin{array}{l}\text { univoque } \\
\text { univoque }\end{array}$} \\
\hline$n=2$ & Root bigger than 2 & & \\
\hline$n=3$ & $111(110)^{\infty}$ & $111(110)^{\infty}$ & \\
\hline$n=4$ & $111(0110)^{\infty}$ & $111(0110)^{\infty}$ & \\
\hline$n=3 k+1$ & $111\left(0(000)^{k-1} 110\right)^{\infty}$ & $11(011)^{k-1} 1\left((011)^{k} 0\right)^{\infty}$ & \\
\hline$n=3 k+2$ & $111\left(00(000)^{k-1} 110\right)^{\infty}$ & $11(011)^{k-1} 1001(101)^{k-1} 0111\left(11(011)^{k-1} 110\right)^{\infty}$ & \\
\hline$n=3 k+3$ & $111\left((000)^{k} 110\right)^{\infty}$ & $11(011)^{k} 1(110)^{\infty}$ & \\
\hline \multicolumn{4}{|c|}{$P_{P_{\psi_{2}}(x) x^{n}+B_{\psi_{2}}(x)}$} \\
\hline$n=1$ & 10001 & $0000(1)^{\infty}$ & \multirow{3}{*}{ periodic self-bracketed } \\
\hline$n=2$ & 11 & $0(1)^{\infty}$ & \\
\hline$n=3$ & 11001010011 & $(1011110)^{\infty}$ & \\
\hline$n=4$ & 11010011001011 & $110100(10111111)^{\infty}$ & \multirow{4}{*}{$\begin{array}{l}\text { periodic self-bracketed } \\
\text { periodic self-bracketed } \\
\text { periodic self-bracketed }\end{array}$} \\
\hline$n=3 k+1$ & $1(101)^{k} 00(110)^{k} 0(101)^{k} 1$ & $\left(1(101)^{k} 00(110)^{k} 0(101)^{k} 0\right)^{\infty}$ & \\
\hline$n=3 k+2$ & $1(101)^{k} 1$ & $\left(1(101)^{k} 0\right)^{\infty}$ & \\
\hline$n=3 k+3$ & $(110)^{k+1} 0(101)^{k+1} 001(101)^{k} 1$ & $\left(110(110)^{k} 0(101)^{k+1} 001(101)^{k} 0\right)^{\infty}$ & \\
\hline \multicolumn{4}{|c|}{$P_{\psi_{2}}(x) x^{n}-B_{\psi_{2}}(x)$} \\
\hline$n=1$ & Root bigger than 2 & & \multirow{7}{*}{$\begin{array}{l}\text { periodic self-bracketed } \\
\text { periodic self-bracketed } \\
\text { periodic self-bracketed }\end{array}$} \\
\hline$n=2$ & 11111 & $(11110)^{\infty}$ & \\
\hline$n=3$ & 111011 & $(111010)^{\infty}$ & \\
\hline$n=4$ & 1110011 & $(1110010)^{\infty}$ & \\
\hline$n=3 k+1$ & $11100(000)^{k-1} 11$ & $\left(11(011)^{k-1} 1001(101)^{k-1} 0\right)^{\infty}$ & \\
\hline$n=3 k+2$ & $111(000)^{k} 11$ & $\left(11(011)^{k} 11(101)^{k} 0\right)^{\infty}$ & \\
\hline$n=3 k+3$ & $1110(000)^{k} 11$ & $\left(11(011)^{k}(101)^{k+1} 0\right)^{\infty}$ & \\
\hline
\end{tabular}


Proof. Table 4 as well as Table 5 later on, are the results of a computation. The results themselves are easy to verify, so the main interest is the process that the computer went through to discover these results. This is the subject of Section 7 We also list which of these numbers correspond to periodic self-bracketed sequences for completeness.

This lemma gives an easy corollary

Corollary 5.1. There exists a neighborhood $\left[\psi_{2}-\varepsilon, \psi_{2}+\varepsilon\right]$ that contains no univoque numbers.

In fact we will see in Section 5.3 that this is actually quite a large neighborhood. This is probably also true for other $\psi_{r}$, where the neighborhood would not be nearly as large.

5.2. The limit point $\chi$. We know that $\chi$ is a limit point of the set of Pisot numbers. Moreover, we know exactly what the sequences tending to $\chi$ look like. Let $P_{\chi}(x)=x^{4}-x^{3}-2 x^{2}+1$ be the Pisot polynomial associated with $\chi$. Let $A_{\chi}(x)=x^{3}+x^{2}-x-1$ and $B_{\chi}(x)=x^{4}-x^{2}+1$ be two polynomials associated with $P_{\chi}(x)$. Then for sufficiently large $n$, the polynomials $P_{\chi}(x) x^{n} \pm A_{\chi}(x)$ and $P_{\chi}(x) x^{n} \pm B_{\chi}(x)$ admit a unique root between 1 and 2 , which is a Pisot number. See for example [6, 14.

Moreover, we have that the roots of $P_{\chi}(x) x^{n}-A_{\chi}(x)$ and $P_{\chi}(x) x^{n}-B_{\chi}(x)$ approach $\chi$ from above, and those of $P_{\chi}(x) x^{n}+A_{\chi}(x)$ and $P_{\chi}(x) x^{n}+B_{\chi}(x)$ approach $\chi$ from below. This follows as $P_{\chi}(1)=-1$ and $P_{\chi}(2)=1$, with $P_{\chi}(x)$ strictly increasing on $[1,2]$, along with the fact that on $(1,2]$ we have $A_{\chi}(x), B_{\chi}(x)>0$. Although we need only examine the sequences of Pisot numbers approaching $\chi$ from below, we give the results for all sequences approaching $\chi$ for completeness.

Lemma 5.2. The greedy and lazy expansions of Pisot numbers approaching $\chi$ are summarized in Table 5 .

Lemma 5.2 above, along with Proposition 3.1 and Corollary 5.1, prove the following result:

Theorem 5.2. There are only a finite number of univoque Pisot numbers less than $\chi$.

In addition, Lemma 5.2 proves the result

Theorem 5.3. The univoque Pisot number $\chi$ is the smallest limit point of univoque Pisot numbers. It is a limit point from above of regular univoque Pisot numbers.

5.3. Univoque Pisot numbers less than $\chi$. Our goal in this section is to describe our search for univoque Pisot numbers below the first limit point $\chi$. We know that all univoque Pisot numbers less than $\chi$ are either in the range $\left[\kappa, \psi_{2}\right]$, or in the range $\left[\pi_{2}, \chi\right]$. Here $\kappa$ is the Komornik-Loreti constant (approximately 1.787231), $\psi_{2}$ is approximately $1.839286755, \pi_{2}>1.8705$ and $\chi$ is approximately 1.905166168 . We will search for Pisot numbers in the range $[1.78,1.85]$ and $[1.87,1.91]$.

To use the algorithm of Boyd [10, we need to do an analysis of the limit points in these two ranges. In particular, we need to do an analysis of the limit points $\psi_{2}$ and $\chi$. 
TABLe 5. Greedy and lazy expansion for regular Pisot numbers approaching $\chi$.

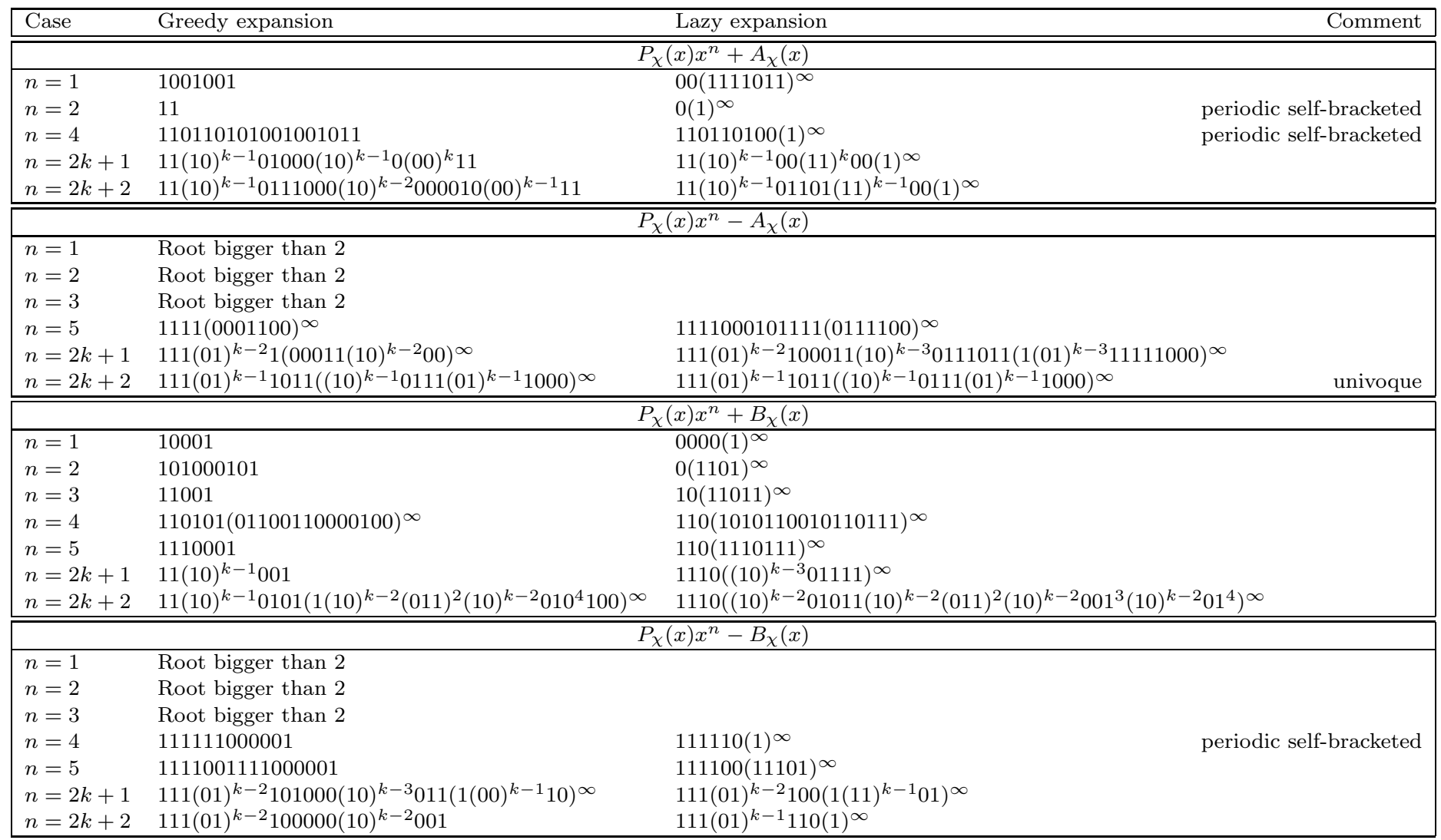


We use the notation of [10]. Let $P(z)$ be a minimal polynomial of degree $s$ of a Pisot number $\theta$, and $Q(z)=z^{s} P(1 / z)$ be the reciprocal polynomial. Let $A(z)$ be a second polynomial with integer coefficients, such that $|A(z)| \leq|Q(z)|$ for all $|z|=1$. Then $f(z)=A(z) / Q(z)=u_{0}+u_{1} z+u_{2} z^{2}+\cdots \in \mathbb{Z}[[z]]$ is a rational function associated with $\theta$. The sign of $A(z)$ is chosen in such a way that $u_{0} \geq 1$. Then by Dufresnoy and Pisot [19] we have the following:

$$
\begin{aligned}
1 & \leq u_{0} \\
u_{0}^{2}-1 & \leq u_{1} \\
w_{n}\left(u_{0}, \cdots, u_{n-1}\right) & \leq u_{n} \leq w_{n}^{*}\left(u_{0}, \cdots, u_{n-1}\right)
\end{aligned}
$$

where $w_{n}$, and $w_{n}^{*}$ are defined below. Let $D_{n}(z)=-z^{n}+d_{1} z^{n-1}+\cdots+d_{n}$ and $E_{n}(z)=-z^{n} D_{n}(1 / z)$. Solve for $d_{1}, \cdots, d_{n}$ such that

$$
\frac{D_{n}(z)}{E_{n}(z)}=u_{0}+u_{1} z+\cdots+u_{n-1} z^{n-1}+w_{n}\left(u_{0}, \cdots, u_{n-1}\right) z^{n}+\cdots
$$

This will completely determine $w_{n}$. There are some nice recurrences for $w_{n}$ and $D_{n}$, which simplify the computation of $w_{n}$ [10]. We have that $w_{n}^{*}$ is computed very similarly, instead considering $D_{n}^{*}(z)=z^{n}+d_{1} z^{n-1}+\cdots+d_{n}$ and $E_{n}^{*}(z)=z^{n} D_{n}(1 / z)$. Expansions $u_{0}+u_{1} z+\cdots$ satisfying Equation (11) with integer coefficients are in a one-to-one correspondence with Pisot numbers.

Using this notation, Lemma 2 of [10] becomes:

Lemma 5.3. Let $f=u_{0}+u_{1} z+u_{2} z^{2}+\cdots$ be associated with a limit point $\theta$ in $S^{\prime}$. Suppose that $w_{N}^{*}-w_{N} \leq 9 / 4$ for some $N$. Then for any $n \geq N$, there are exactly two $g$ with expansions beginning with $u_{0}+u_{1} z+\cdots+u_{n-1} z^{n-1}$. Moreover, for all $n \geq N$, all $g$ beginning with $u_{0}+u_{1} z+\cdots+u_{n-1} z^{n-1}$ are associated with the regular Pisot numbers approaching the limit point $\theta$.

So in particular, we need to find the expansion of the limit points around $\psi_{2}$ and $\chi$ : Consider the following rational functions associated with the limit points $\psi_{2}$ and $\chi$ :

(1) Consider

$$
-\frac{x+1}{x^{3}+x^{2}+x-1}=1+2 x+3 x^{2}+6 x^{3}+11 x^{4}+20 x^{5}+37 x^{6}+\cdots
$$

as the first of the two rational functions associated with the limit point $\psi_{2}$.

A quick calculation shows that $w_{24}^{*}-w_{24}<9 / 4$.

(2) Consider

$$
\frac{x^{3}-1}{x^{3}+x^{2}+x-1}=1+x+2 x^{2}+3 x^{3}+6 x^{4}+11 x^{5}+20 x^{6}+\cdots
$$

as the second of the two rational functions associated with the limit point $\psi_{2}$. A quick calculation shows that $w_{11}^{*}-w_{11}<9 / 4$.

(3) Consider

$$
-\frac{x^{3}+x^{2}-x-1}{x^{4}-2 x^{2}-x+1}=1+2 x+3 x^{2}+6 x^{3}+11 x^{4}+21 x^{5}+40 x^{6}+\cdots
$$

as the first of the two rational functions associated with the limit point $\chi$. A quick calculation shows that $w_{33}^{*}-w_{33}<9 / 4$. 
(4) Consider

$$
\frac{x^{4}-x^{2}+1}{x^{4}-2 x^{2}-x+1}=1+x+2 x^{2}+4 x^{3}+8 x^{4}+15 x^{5}+29 x^{6}+\cdots
$$

as the second of the two rational functions associated with the limit point $\chi$. A quick calculation shows that $w_{44}^{*}-w_{44}<9 / 4$.

Using this result, we were able to use Boyd's algorithm for finding Pisot numbers in the two ranges $[1.78,1.85]$ and $[1.87,1.91]$ (which contain $\left[\kappa, \psi_{2}\right]$ and $\left[\pi_{2}, \chi\right]$ ), where when we have an expansion that matches one of the four rational functions listed above, we prune that part of the search tree, as we would only find regular Pisot numbers of a known form.

There were 227 Pisot numbers in the first range (minus the known regular Pisot numbers pruned by the discussion above), and 303 in the second range (similarly pruned). There were 530 such Pisot numbers in total.

A corollary of this computation worth noting is

\section{Corollary 5.2.}

- The only Pisot numbers in $\left[\psi_{2}-10^{-8}, \psi_{2}+10^{-8}\right]$ are $\psi_{2}$ and the regular Pisot numbers associated with $\psi_{2}$.

- The only Pisot numbers in $\left[\chi-10^{-13}, \chi+10^{-3}\right]$ are $\chi$ and the regular Pisot numbers associated with $\chi$.

We then checked each of these 530 Pisot numbers to see if they were univoque. We did this by computing the greedy and lazy $\beta$-expansion of each Pisot number and checked if they were equal. This calculation gave the following theorem:

Theorem 5.4. There are exactly two univoque Pisot numbers less than $\chi$. They are

- $1.880000 \cdots$ the root in $(1,2)$ of the polynomial $x^{14}-2 x^{13}+x^{11}-x^{10}-$ $x^{7}+x^{6}-x^{4}+x^{3}-x+1$ with univoque expansion $111001011(1001010)^{\infty}$.

- $1.886681 \cdots$ the root in $(1,2)$ of the polynomial $x^{12}-2 x^{11}+x^{10}-2 x^{9}+$ $x^{8}-x^{3}+x^{2}-x+1$ with univoque expansion $111001101(1100)^{\infty}$.

\section{Regular Pisot numbers associated With $\psi_{r}$}

The goal of this section is to show that 2 is the limit point of univoque Pisot numbers. We will do this by observing that for each $r$, there are regular Pisot numbers between $\psi_{r}$ and 2 that are univoque. We know that the $\psi_{r}$ are limit points of the set of regular Pisot numbers. Moreover we know that $\psi_{r} \rightarrow 2$ as $r \rightarrow \infty$. Using the notation of Section 5.1 we define $P_{\psi_{r}}$ and $A_{\psi_{r}}$. We denote the Pisot number associated with the polynomial $P_{\psi_{r}}(x) x^{n}-A_{\psi_{r}}(x)$, as $\psi_{r, n}^{A,-}$.

Theorem 6.1. Let $n \geq r+1$. Then the greedy expansion of $\psi_{r, n}^{A,-}$ is

$$
1^{r+1}\left(0^{n-r-1} 1^{r} 0\right)^{\infty}
$$


Proof. First we expand this expansion to see that it is equivalent to

$$
\begin{aligned}
& 1=\frac{1}{x}+\cdots+\frac{1}{x^{r+1}}+\left(0+\frac{1}{x^{n+1}}+\cdots+\frac{1}{n+r}+0\right)\left(\frac{1}{1-\frac{1}{x^{n}}}\right) \\
& \Longrightarrow \quad 1=\frac{\frac{1}{x}-\frac{1}{x^{r+2}}}{1-\frac{1}{x}}+\left(\frac{\frac{1}{x^{n+1}}-\frac{1}{x^{n+r+1}}}{1-\frac{1}{x}}\right)\left(\frac{1}{1-\frac{1}{x^{n}}}\right) \\
& \begin{aligned}
\left(1-\frac{1}{x}\right)\left(1-\frac{1}{x^{n}}\right) & =\left(\frac{1}{x}-\frac{1}{x^{r+2}}\right)\left(1-\frac{1}{x^{n}}\right)+\left(\frac{1}{x^{n+1}}-\frac{1}{x^{n+r+1}}\right) \\
\Longrightarrow \quad x^{r+2}(x-1)\left(x^{n}-1\right) & =x\left(x^{r+1}-1\right)\left(x^{n}-1\right)+x^{r+2}-x^{2}
\end{aligned}
\end{aligned}
$$

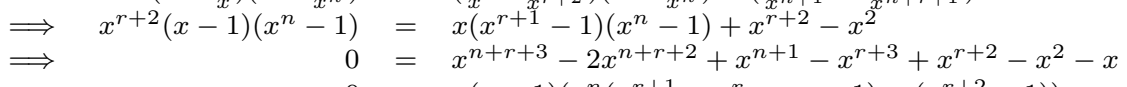

$$
\begin{aligned}
& \Longrightarrow \quad 0=x(x-1)\left(x^{n}\left(x^{r+1}-x^{r}-\cdots-1\right)-\left(x^{r+2}-1\right)\right) \\
& \Longrightarrow \quad 0=x(x-1)\left(P_{\psi_{r}}(x) x^{n}-A_{\psi_{r}}(x)\right) \text {. }
\end{aligned}
$$

So we see that this is a valid expansion for this regular Pisot number.

To observe that this is indeed the greedy $\beta$-expansion we observe that the $\beta$ expansion starts with $r+1$ consecutive 1's, and all strings of consecutive 1's after this are shorter than $r+1$. Hence it follows from Theorem 2.1.

By Corollary 2.1 we get the immediate result:

Corollary 6.1. If $n \geq 2(r+1)$ the regular Pisot number $\psi_{r, n}^{A,-}$ is not univoque.

So, the main theorem is

Theorem 6.2. Assume $r+1 \leq n<2(r+1)$. Then $\psi_{r, n}^{A,-}$ is a univoque Pisot number.

Proof. So it suffices to see that the equation (2) is both greedy and lazy. This follows from Theorems 2.1 and 2.2 .

Corollary 6.2. We have that 2 is a limit point of $S \cap \mathcal{U}$.

Proof. We see that $\psi_{r, n}^{A,-}$ is always greater than $\psi_{r}$. Further, for $r+1 \leq n \leq 2(r+1)$ we have that $\psi_{r, n}^{A,-}$ is less than 2 , which follows from noticing that $P_{\psi_{r}}(1) 1^{n}-$ $A_{\psi_{r}}(1)=(1-1-1-\cdots-1)-\left(1^{r+1}-1\right)<0$ and

$$
P_{\psi_{r}}(2) 2^{n}-A_{\psi_{r}}(2)=2^{n}\left(2^{r+1}-2^{r}-\cdots-1\right)-\left(2^{r+1}-1\right)=2^{n}-2^{r+1}+1>0 .
$$

Further, we see that $\psi_{r}$ tends to 2 .

\section{Automated conjectures And PRoofs}

The results in Tables 4 and 5 were generated automatically. This section describes the algorithms that were used to do this.

- Computing the greedy $\beta$-expansion. We will explain, given $\beta$ a root of $P_{\beta}(x)$, how to compute the greedy $\beta$-expansion of 1 (assuming periodicity).

- Computing the lazy $\beta$-expansion. We will explain, given $\beta$ a root of $P_{\beta}(x)$, how to compute the lazy $\beta$-expansion of 1 (assuming periodicity).

- Creating the conjecture. We will explain how with the greedy or lazy $\beta$-expansion of 1 for a sequence of regular Pisot numbers, how to create a conjecture of the general pattern of the $\beta$-expansion.

- Verifying conjecture. We will explain how a general pattern can be verified to be a valid $\beta$-expansion.

- Check greedy/lazy/univoque/periodic self-bracketed expansion. We will explain how to check if a general pattern is a valid greedy, lazy, univoque or periodic self-bracketed $\beta$-expansion. 
7.1. Computing the greedy $\beta$-expansion. The greedy algorithm does the most work possible at any given step (see the discussion in Section 2.2.1).

The computation is done symbolically modulo the minimal polynomial of $\beta$, and floating point numbers are used only when computing $x_{n}$. A check is done on $\beta r_{n-1}-x_{n}$ to ensure that the calculation is being done with sufficient digits to guarantee the accuracy of the result.

A list of previously calculated $r_{n}$ 's is kept and checked upon each calculation to determine when the $\beta$-expansion becomes eventually periodic.

7.2. Computing the lazy $\beta$-expansion. Basically, the algorithm tries to do the minimal work at any given time (see discussion in Section 2.2.2).

As with the greedy expansion, computations are done as a mixture of floating point and symbolic, to allow for recognition of periodicity, with the same checks being performed as before to ensure the accuracy of the result.

7.3. Creating the conjecture. In this section we will explain how, given $d_{q_{1}}(1)$ and $d_{q_{2}}(1)$ (or the related lazy $\beta$-expansions), for some "regular sequence" of Pisot numbers $q_{k}$, we can conjecture a "nice" expression for $d_{q_{k}}(1)$. This is probably best done by example. Assume that two consecutive greedy expansions give the finite expansions:

$$
\begin{aligned}
& d_{q_{1}}(1)=0011011011 \\
& d_{q_{2}}(1)=00111101101011 .
\end{aligned}
$$

We start by reading characters from each string into the "string read" expression:

\begin{tabular}{lll} 
String 1 & String 2 & String read \\
\hline 001101101011 & 0011110110101011 & empty \\
01101101011 & 011110110101011 & 0 \\
1101101011 & 11110110101011 & 00 \\
101101011 & 1110110101011 & 001 \\
01101011 & 110110101011 & 0011
\end{tabular}

At this point we see that the next characters to read from String 1 and String 2 are different. We use a result that is only observed computationally, and has no theoretical reason for being true. This is that the size of every part that depends on the value of $k$ is of the same size, which is known before the computation begins. So an expression $d_{q_{k}}(1)=v_{1}\left(w_{1}\right)^{k} v_{2}\left(w_{2}\right)^{k} \cdots$ would have all $\left|w_{i}\right|$ constant, and known in advance. In this case, we are assuming that this size is 2 . So we check if the next two characters of String 2 are the same as the previous two characters of String 1. (In this case, both of these are "11".) We then truncate the result to give something of the form $(11)^{k}$ which is valid for both strings.

So we continue:

\begin{tabular}{lll} 
String 1 & String 2 & String read \\
\hline 01101011 & 110110101011 & 0011 \\
01101011 & 0110101011 & $00(11)^{k}$ \\
1101011 & 110101011 & $00(11)^{k} 0$ \\
$\vdots$ & $\vdots$ & $\vdots$ \\
1 & 011 & $00(11)^{k} 0110101$
\end{tabular}

Again we check if the next two characters of String 2 are equal to the previous two characters in String 1. We also notice that the two characters "01" are in fact 
repeated more times than this, so we get

\begin{tabular}{lll} 
String 1 & String 2 & String read \\
\hline 1 & 011 & $00(11)^{k} 0110101$ \\
1 & 1 & $00(11)^{k} 01101(01)^{k}$ \\
1 & 1 & $00(11)^{k} 011(01)^{k+1}$ \\
empty & empty & $00(11)^{k} 011(01)^{k+1} 1$
\end{tabular}

So we would conjecture that $d_{q_{k}}(1)=00(11)^{k} 011(01)^{k+1} 1$.

It should be pointed out that this is in no way a proof that this is the general result. This has to be done separately in Sections 7.4 and 7.5 .

7.4. Verifying conjecture. In this section we show, given a conjectured expansion for $q_{k}$, how we would verify that this is a valid expansion for all $q_{k}$. It should be noticed that this does not prove what type of $\beta$-expansion it is (i.e., greedy, lazy, ...). This will be done in Section 7.5.

We will demonstrate this method, by considering an example. Consider the greedy expansion $d_{\beta_{k}}(1)=1(101)^{k} 1=11(011)^{k}$ associated with the greedy expansion of 1 for the Pisot root associated with

$$
P_{k}^{*}(x)=P_{\psi_{2}}(x) x^{3 k+2}+B_{\psi_{2}}(x)=\left(x^{3}-x^{2}-x-1\right) x^{3 k+2}+(x+1) .
$$

For convenience we write $\beta$ for this root (where $\beta$ will depend on $k$ ). We then see that this expansions implies

$$
\frac{1}{\beta}+\frac{1}{\beta^{2}}+\frac{1}{\beta^{4}}+\frac{1}{\beta^{5}}+\frac{1}{\beta^{7}}+\frac{1}{\beta^{8}}+\cdots+\frac{1}{\beta^{3 k+1}}+\frac{1}{\beta^{3 k+2}}=1 .
$$

This simplifies to

$$
\beta^{-1}+\beta^{-2}+\left(\beta^{-4}+\beta^{-5}\right) \sum_{j=0}^{k-1}\left(\beta^{3 j}\right)^{-1}=1 .
$$

By subtracting 1 from both sides, and clearing the denominator, this is equivalent to $D_{k}(\beta)=0$ where

$$
D_{k}(x):=-x^{3 k+5}+x^{3 k+4}+x^{3 k+3}+x^{3 k+2}-x-1 .
$$

But we notice that

$$
D_{k}(x)=-\left(P_{\psi_{2}}(x) x^{3 k+2}+B_{\psi_{2}}(x)\right)
$$

hence $D_{k}(x)=-P_{k}^{*}(x)$. All of these processes can be automated. The hardest part is finding a co-factor $C_{k}(x)$ such that $D_{k}(x)=C_{k}(x) P_{k}^{*}(x)$. (We are not always so lucky that $C_{k}(x)=-1$ as was the case in this example.) Here we noticed computationally that $C_{k}(x)$ is always of the form:

$$
C_{k}(x)=a_{n} x^{b_{n} k+c_{n}}+a_{n-1} x^{b_{n-1} k+c_{n-1}}+\cdots+a_{2} x^{b_{2} k+c_{2}}+a_{1} x^{b_{1} k+c_{1}} .
$$

For our purposes it was unnecessary to prove that this is always the case, as we could easily verify it for all cases checked, and we were using this as a tool to verify the conjectured general form. 
7.5. Checking the greedy/lazy/univoque/periodic self-bracketed $\beta$-expansion. In this section we discuss how one would check if an expression (conjectured using the techniques of Section 7.3 and verified as a $\beta$-expansion in Section 7.4) is in fact a greedy, lazy or periodic self-bracketing $\beta$-expansion. Consider a general expression of the form

$$
E(k):=v_{1}\left(w_{1}\right)^{k} v_{2}\left(w_{2}\right)^{k} \cdots\left(w_{n-1}\right)^{k} v_{n}\left(u_{1}\left(w_{n}\right)^{k} \cdots\left(w_{n+m}\right)^{k} u_{m}\right)^{\infty}
$$

where the $w_{i}$ all have the same length (this is in fact the case for all problems that we studied). Then the main thing to notice is that there exists a $K$ such that if the $\beta$-expansion $E(K)$ has a desired property (either being or not being greedy, lazy, etc.), then for all $k \geq K$ we have that $E(k)$ has the same property. Moreover the $K$ is explicitly computable, being a function of the lengths of the $v_{i}, w_{i}$ and $u_{i}$. This means that what initially looks like an infinite number of calculations is in fact a finite number of calculations. The way to see this is that for sufficiently large $k$, most of the comparisons will be done between the $w_{i}$ 's, and then an increase in $k$ will not change this, but just add another redundant check to something already known.

\section{Comments, OPEn QUestions AND FURTher WORK}

There are some interesting observations that can be made from the data and results so far. This investigation has opened up a number of questions.

- First, given a sequence of greedy or lazy $\beta$-expansions of a nice sequence of Pisot numbers $q_{k}$ that looks like

$$
E(k):=v_{1}\left(w_{1}\right)^{k} v_{2}\left(w_{2}\right)^{k} \cdots\left(w_{n-1}\right)^{k} v_{n}\left(u_{1}\left(w_{n}\right)^{k} \cdots\left(w_{n+m}\right)^{k} u_{m}\right)^{\infty},
$$

is it always true that $\left|w_{1}\right|=\left|w_{2}\right|=\cdots=\left|w_{n+m}\right|$ ?

- Is the co-factor from Section 7.4 always of the form

$$
C_{k}(x)=a_{n} x^{b_{n} k+c_{n}}+a_{n-1} x^{b_{n-1} k+c_{n-1}}+\cdots+a_{2} x^{b_{2} k+c_{2}}+a_{1} x^{b_{1} k+c_{1}} ?
$$

- It appears in Table 3 that for all Salem numbers of degree 4 and 6 greater than $\approx 1.83$, these Salem numbers are univoque. Is this just an artifact of small degrees, or is something more general going on?

- In general, are the greedy/lazy $\beta$-expansions even periodic for Salem numbers? (This is not known to be true; see [15] for more details.)

- It is known that Pisot numbers can be written as a limit of Salem numbers, where if $P(x)$ is the minimal polynomial of a Pisot number, then $P(x) x^{n} \pm$ $P^{*}(x)$ has a Salem number as a root, which tends to the root of the Pisot number. Some preliminary and somewhat haphazard investigation suggests that we might be able to find a "regular" looking expression for the greedy (resp. lazy) $\beta$-expansion of these Salem numbers, which tends towards the greedy (resp. lazy) $\beta$-expansion of the Pisot number. If true, this could have implications towards questions concerning the $\beta$-expansions of Salem numbers being eventually periodic.

\section{Note AdDED on June 12, 2006}

Just before submitting this paper we came across a paper where the topological structure of the set $\mathcal{U}$ and of its (topological) closure are studied. We cite it here for completeness: 
V. Komornik, P. Loreti, On the structure of univoque sets, J. Number Theory, 122 (2007), $157-183$

One can also read consequences of the results of that paper in

M. de Vries, Random $\beta$-expansions, unique expansions and Lochs' Theorem, Ph.D. Thesis, Vrije Universiteit Amsterdam, 2005.

(available at http://www.cs.vu.nl/ mdvries/proefschrift.pdf).

\section{ACKNOWLEDGMENTS}

The authors wish to thank David Boyd for stimulating discussions, and the referee for a careful reading of the manuscript.

\section{REFERENCES}

[1] J.-P. Allouche, Théorie des Nombres et Automates, Thèse d'État, Bordeaux, 1983.

[2] J.-P. Allouche, M. Cosnard, Itérations de fonctions unimodales et suites engendrées par automates, C. R. Acad. Sci. Paris, Sér. 1296 (1983) 159-162. MR693191 (85f:58082b)

[3] J.-P. Allouche, M. Cosnard, The Komornik-Loreti constant is transcendental, Amer. Math. Monthly 107 (2000) 448-449. MR 1763399

[4] J.-P. Allouche, M. Cosnard, Non-integer bases, iteration of continuous real maps, and an arithmetic self-similar set, Acta Math. Hung. 91 (2001) 325-332. MR1912007 (2003f:11013)

[5] J.-P. Allouche, J. Shallit, The ubiquitous Prouhet-Thue-Morse sequence, in C. Ding, T. Helleseth and H. Niederreiter (Eds.) Sequences and their applications, Proceedings of SETA'98, Springer, 1999, pp. 1-16. MR.1843077 (2002e:11025)

[6] M. Amara, Ensembles fermés de nombres algébriques, Ann. Sci. École Norm. Sup. 83 (1966) 215-270. MR0237459 (38:5741)

[7] M.-J. Bertin, A. Descomps-Guilloux, M. Grandet-Hugot, M. Pathiaux-Delefosse, J.-P. Schreiber, Pisot and Salem numbers, Birkhäuser, 1992. MR.1187044 (93k:11095)

[8] A. Bertrand, Développements en base de Pisot et répartition modulo 1, C. R. Acad. Sci. Paris, Sér. A-B 285 (1977) 419-421. MR0447134 (56:5449)

[9] P. Borwein, Computational excursions in analysis and number theory, CMS Books in Mathematics/Ouvrages de Mathématiques de la SMC, 10, Springer-Verlag, New York, 2002. MR.1912495 (2003m:11045)

[10] D. W. Boyd, Pisot and Salem numbers in intervals of the real line, Math. Comp. 32 (1978) 1244-1260. MR 0491587 (58:10812)

[11] D. W. Boyd, Pisot numbers in the neighbourhood of a limit point, I, J. Number Theory 21 (1985) 17-43. MR804914 (87c:11096a)

[12] D. W. Boyd, Pisot numbers in the neighborhood of a limit point, II, Math. Comp. 43 (1984) 593-602. MR758207 (87c:11096b)

[13] D. W. Boyd, Salem numbers of degree four have periodic expansions, in J.-H. De Coninck, C. Levesque (Eds.), Théorie des Nombres, Québec, 1987, Walter De Gruyter, 1989, pp. 57-64. MR:1024551 (90j:11071)

[14] D. W. Boyd, On beta expansions for Pisot numbers, Math. Comp. 65 (1996) 841-860. MR:1325863 (96g:11090)

[15] D. W. Boyd, On the beta expansion for Salem numbers of degree 6, Math. Comp. 65 (1996) 861-875, S29-S31. MR1333306 (96g:11091)

[16] K. Dajani and C. Kraaikamp, From greedy to lazy expansions and their driving dynamics, Expo. Math. 20 (2002) 315-327. MR.1940010(2003h:11089)

[17] Z. Daróczy, I. Kátai, Univoque sequences, Publ. Math. Debrecen 42 (1993) 397-407. MR1229687 (94i:11011)

[18] Z. Daróczy, I. Kátai, On the structure of univoque numbers, Publ. Math. Debrecen 46 (1995) 385-408. MR:1336377 (96h:11006)

[19] J. Dufresnoy, Ch. Pisot, Étude de certaines fonctions méromorphes bornées sur le cercle unité. Application à un ensemble fermé d'entiers algébriques, Ann. Sci. École Norm. Sup. 72 (1955) 69-92. MR.0072902 (17:349d)

[20] P. Erdős, I. Joó, V. Komornik, Characterization of the unique expansions $1=\sum_{i=1}^{\infty} q^{-n_{i}}$, and related problems, Bull. Soc. Math. France 118 (1990) 377-390. MR.1078082 (91j:11006) 
[21] P. Glendinning and N. Sidorov, Unique representations of real numbers in non-integer bases, Math. Res. Letters 8 (2001) 447-472. MR.1851269(2002i:11009)

[22] V. Komornik, P. Loreti, Unique developments in non-integer bases, Amer. Math. Monthly 105 (1998) 636-639. MR1633077 (99k:11017)

[23] V. Komornik, P. Loreti, A. Pethő, The smallest univoque number is not isolated, Publ. Math. Debrecen 62 (2003) 429-435. MR2008106 (2005b:11010)

[24] M. Lothaire, Algebraic combinatorics on words, Cambridge University Press, 2002. MR.1905123 (2003i:68115)

[25] R. C. Lyndon and M. P. Schützenberger, The equation $A^{M}=b^{N} c^{P}$ in a free group, Michigan Math. J. 9 (1962) 289-298. MR0162838(29:142)

[26] W. Parry, On the $\beta$-expansions of real numbers, Acta Math. Acad. Sci. Hungar. 11 (1960) 401-416. MR0142719 (26:288)

[27] A. Rényi, Representations for real numbers and their ergodic properties, Acta Math. Acad. Sci. Hungar. 8 (1957) 477-493. MR0097374(20:3843)

[28] R. Salem, Power series with integral coefficients, Duke Math. J. 12 (1945) 153-172. MR0011720 (6:206b)

[29] K. Schmidt, On periodic expansions of Pisot and Salem numbers, Bull. London Math. Soc. 12 (1980) 269-278. MR576976 (82c:12003)

[30] F. L. Talmoudi, Sur les nombres de $S \cap[1,2]$, C. R. Acad. Sci. Paris, Sér. Math. 285 (1977) 969-971. MR507210 (80c:12003)

[31] F. L. Talmoudi, Sur les nombres de $S \cap[1,2[$, C. R. Acad. Sci. Paris, Sér. Math. 287 (1978) 739-741. MR516773(80a:12004)

CNRS, LRI, BÂtiment 490, Université Paris-Sud, 91405 Orsay Cedex, France

E-mail address: allouche@lri.fr

Liafa, CNRS UMr 7089, 2 Place Jussieu, 75251 Paris Cedex 05, France, and UniverSITÉ PARIS 8

E-mail address: Christiane.Frougny@liafa.jussieu.fr

Department of Pure Mathematics, University of Waterloo, Waterloo, Ontario, CANADA N2L 3G1

E-mail address: kghare@math.uwaterloo.ca 